\title{
Calcium and Vitamin D Supplementation and Cognitive Impairment in the Women's Health Initiative
}

\author{
Dr. Rebecca C. Rossom, MD, MSCR, Dr. Mark A. Espeland, PhD, Dr. JoAnn E. Manson, MD, \\ DrPH, Dr. Maurice W. Dysken, MD, Dr. Karen C. Johnson, MD, MPH, Dr. Dorothy S. Lane, \\ MD, MPH, Dr. Erin S. LeBlanc, MD, MPH, Dr. Frank A. Lederle, MD, Dr. Kamal H. Masaki, \\ MD, and Dr. Karen L. Margolis, MD, MPH \\ Health Partners Research Foundation, University of Minnesota, Minneapolis, MN (Dr. Rossom \\ and Dr. Margolis); Division of Public Health Sciences, Wake Forest University Health Sciences, \\ Winston-Salem, NC (Dr. Espeland); Division of Preventive Medicine, Brigham and Women's \\ Hospital, Harvard Medical School, Boston, MA (Dr. Manson); Geriatric Research Education and \\ Clinical Center, Minneapolis VA Health Care System, Minneapolis, MN (Dr. Dysken); Department \\ of Preventive Medicine, University of Tennessee Health Science Center, Memphis, TN (Drs. \\ Johnson); Department of Preventive Medicine, Stony Brook University School of Medicine, Stony \\ Brook, NY (Dr. Lane); School of Medicine, Oregon Health \& Science University, Portland, OR (Dr. \\ LeBlanc); Center for Chronic Disease Outcomes Research, Minneapolis VA Health Care System, \\ Minneapolis, MN (Dr. Lederle); Department of Geriatric Medicine, John A. Burns School of \\ Medicine, University of Hawaii, Manoa, HI (Dr. Masaki)
}

\section{Abstract}

Background-Calcium and vitamin D are thought to play important roles in neuronal functioning. Studies have found associations between low serum vitamin D levels and reduced cognitive functioning, as well as high serum calcium levels and reduced cognitive functioning.

Objectives-To examine the effects of vitamin D and calcium on cognitive outcomes in elderly women.

Design-Post-hoc analysis of a randomized double-blinded placebo-controlled trial.

Setting—40 Women's Health Initiative clinical centers across the U.S.

Participants-4143 women aged 65 years and older without probable dementia at baseline who participated in the WHI Calcium and Vitamin D trial and the Women's Health Initiative Memory Study.

Interventions-2034 women were randomized to $1000 \mathrm{mg}$ of calcium carbonate combined with $400 \mathrm{IU}$ of vitamin $\mathrm{D}_{3} ; 2109$ women were randomized to placebo.

Measurements-Primary: classifications of probable dementia or mild cognitive impairment via a 4-phase protocol that included central adjudication. Secondary: global cognitive function and individual cognitive subtests.

Corresponding author: Rebecca Rossom, MD, MSCR. $817033^{\text {rd }}$ Ave South, P.O. Box 1524; MailStop 21111R, Minneapolis, MN, 55440-1524. Rebecca.C.Rossom@ Healthpartners.com. Phone: 952-967-5001. Fax: 952-967-5022.

Author Contributions:

Drs. Rossom and Espeland had full access to all of the data in the study and take responsibility for the integrity of the data and the accuracy of the data analysis.

Rossom, Espeland: study concept and design, analysis and interpretation of data, and preparation of manuscript

Manson, Lederle, Margolis: study concept and design, preparation of manuscript

Dysken, Johnson, Lane, LeBlanc, Masaki: preparation of manuscript 
Results-Mean age of participants was 71 years. During mean follow-up of 7.8 years, there were 39 cases of incident dementia among calcium plus vitamin D subjects compared to 37 cases among placebo subjects (hazard ratio $=1.11,95 \%$ CI: 0.71-1.74, $\mathrm{p}=0.64$ ). Likewise, there were 98 cases of incident mild cognitive impairment among calcium plus vitamin D subjects compared to 108 cases among placebo subjects (hazard ratio $=0.95,95 \%$ CI: $0.72-1.25, \mathrm{p}=0.72$ ). There were no significant differences in incident dementia or mild cognitive impairment, or in global or domainspecific cognitive function between groups.

Conclusion-There was no association between treatment assignment and incident cognitive impairment. Further studies are needed to investigate the effects of vitamin D and calcium separately, on men and in other age and ethnic groups, and with other doses.

\section{Keywords}

Vitamin D; Calcium; Dementia; Cognition; Mild Cognitive Impairment

\section{INTRODUCTION}

Vitamin D and calcium are often taken together, as vitamin D acts to significantly increase calcium absorption in the intestines, and both vitamin $\mathrm{D}$ and calcium are thought to influence neuronal functioning. Vitamin D is thought to protect against neuronal degeneration through a number of mechanisms, including enhancement of antioxidant pathways, increased production of neuron growth factor, and decreased levels of inflammatory biomarkers. ${ }^{1}$ In vitro mouse studies have demonstrated that vitamin D is active in the hippocampus, reversing inflammatory changes related to aging ${ }^{2}$ and protecting against glucocorticoid-induced neuronal death. ${ }^{3}$ However, in a small trial involving human subjects with Alzheimer's disease, high-dose vitamin D2 was not associated with significant changes in cognition or function ${ }^{4}$, and studies of the relationship between 25-

hydroxyvitamin-D $(25(\mathrm{OH}) \mathrm{D})$ levels and cognitive function have reported conflicting results. 5

While calcium is necessary for neuronal functioning, elevated intraneuronal calcium can lead to compromised synaptic function and ultimately neuronal death, and it is becoming apparent that dysregulation of neuronal calcium homeostasis is a hallmark of Alzheimer's pathology. ${ }^{6}$ Disturbances in the calcium homeostasis of Alzheimer's patients may influence the metabolism and production of amyloid-beta peptide and tau, two pathological proteins associated with Alzheimer's disease. ${ }^{7-8}$ Two clinical studies have found inverse associations between serum calcium and cognitive functioning. ${ }^{9-10}$

Cognitive decline in the elderly is a major public health problem, and unfortunately there are currently no known preventative treatments. To our knowledge, no randomized trial has examined the effect of vitamin D and calcium supplementation on cognitive outcomes. We report here the long-term effects of vitamin $\mathrm{D}$ and calcium supplementation on incident dementia, mild cognitive impairment, global cognitive function and domain-specific cognitive function in a large multi-center randomized trial of elderly women.

\section{METHODS}

\section{Participants}

The Women's Health Initiative (WHI) Calcium and Vitamin D $(\mathrm{CaD})$ trial is a large randomized trial designed to determine whether calcium plus vitamin $\mathrm{D}_{3}$ supplementation prevents hip fracture and colorectal cancer in postmenopausal women. ${ }^{11-12}$ Main study results have demonstrated a modest benefit for calcium and vitamin D supplements in preserving bone mass and preventing hip fractures in certain groups, but no benefit in 
prevention of colorectal cancer or other types of fracture. In this manuscript, we report cognition-related outcomes in all $4143 \mathrm{WHI} \mathrm{CaD}$ trial participants who were also enrolled in the Women's Health Initiative Memory Study (WHIMS), an ancillary study to WHI. ${ }^{13-14}$

The eligibility and recruitment methods for the WHIMS and $\mathrm{CaD}$ trials have been described in detail. ${ }^{11,14}$ All WHIMS participants, and thus all participants in this study, were also participants in the WHI Hormone Therapy trials. ${ }^{15}$ Some were also participants in a third trial of dietary modification to compare a low-fat eating pattern to a usual ad-libitum diet. ${ }^{16}$ All participants were 65 years or older and did not have cognitive impairment at baseline. ${ }^{14}$ Major exclusion criteria have been described previously and included those related to diseases associated with significantly increased risk of mortality (invasive cancer in the previous 10 years; any history of breast cancer or a suspicion of breast cancer at the time of screening; acute myocardial infarction, stroke or transient ischemic attacks in the previous 6 months; or known chronic active hepatitis or severe cirrhosis), safety (blood cell counts indicative of disease; severe hypertension; or current use of oral corticosteroids), and adherence or retention (unwillingness or inability to complete baseline study requirements) $)^{11,14}$. Participants were enrolled between 1994 and 1999. Annual cognitive assessments were conducted until the end of the WHI Hormone Therapy trials (in 2002 for the estrogen/progestin trial and 2004 for the estrogen alone trials ${ }^{17-18}$ ). Participants were then asked to re-consent and enroll for clinic-based annual post-trial cognitive assessments, which continued through September, 2007.

In addition, we analyzed the subset of the $1420 \mathrm{CaD}$ participants who also participated in The Women's Health Initiative Study of Cognitive Aging (WHISCA) to examine any effect of randomization to calcium and vitamin D on specific areas of cognitive function. WHISCA is an ancillary study of WHIMS examining whether hormone therapies influence domain-specific cognitive function. WHISCA enrolled 2,304 participants from 14 of the WHIMS clinical sites beginning in September, 1999. Women joined WHISCA an average of 3.0 years after randomization into the WHI Hormone Therapy trials and were followed annually with assessments of eight cognitive domains. ${ }^{19}$ Following the completion of the WHI Hormone Therapy Trials, WHISCA participants were asked to re-consent and enroll in post-trial follow-up to continue the annual cognitive assessments through September, 2007.

\section{Interventions}

Participants in the WHI CaD trial were randomized to receive either two combination tablets containing a total of $1000 \mathrm{mg}$ of calcium carbonate and $400 \mathrm{IU}$ of vitamin $\mathrm{D}_{3}$ per day or identical placebos. These doses were chosen in the early 1990s to evaluate the primary effect of supplementation on hip fracture, with vitamin $\mathrm{D}_{3}$ provided to aid in calcium absorption. Use of personal supplemental calcium up to $1000 \mathrm{mg}$ per day and vitamin D up to $600 \mathrm{IU}$ per day (increased to 1000 IU per day in 1999) was also allowed. ${ }^{20}$

\section{Outcome Assessment}

Participants were assessed via annual clinical visits. The primary outcome measures of WHIMS were classifications of probable dementia, mild cognitive impairment (MCI) and cognitively normal as defined by a protocol that consisted of 4 phases and has been described in detail. ${ }^{14}$ Diagnoses of dementia were based on Diagnostic and Statistical Manual of Mental Disorders, Fourth Edition (DSM-IV) criteria. ${ }^{21}$ Diagnoses of MCI were based on accepted criteria at the time of WHIMS initiation, operationally defined as performance in the $10^{\text {th }}$ or lower percentile on at least 1 CERAD (Consortium to Establish a Registry for Alzheimer's Disease) test based on CERAD norms ${ }^{22}$, a report from the designated informant of functional impairment on the part of the subject that did was not severe enough to interfere with basic activities of daily living, no evidence of a psychiatric 
or medical condition that could account for the cognitive decline, and an absence of adjudicated dementia. ${ }^{23-24}$ Classifications were made by a central adjudication panel of experts at the WHIMS Clinical Coordinating Center at Wake Forest School of Medicine, Winston-Salem, NC.

Secondary outcome measures were global cognitive function as assessed annually using the Modified Mini Mental State Exam (3MSE), ${ }^{25}$ and domain-specific cognitive functions as assessed annually in a subset of $1420 \mathrm{CaD}$ participants via the WHISCA cognitive battery. ${ }^{19}$ The WHISCA cognitive battery included the Digit Span Forward and Backward Test to assess attention and working memory, the Primary Mental Abilities Vocabulary test to assess verbal knowledge, the Card Rotations Test to measure spatial ability, letter and semantic fluency tests to assess verbal fluency, the California Verbal Learning Test to assess verbal memory, the Benton Visual Retention Test to assess short-term figural memory, and the Finger Tapping Test to assess fine motor speed. ${ }^{19}$

Baseline demographic information, medical history and lifestyle variables were primarily obtained via self-report and clinical measurements using standardized study forms as detailed elsewhere. ${ }^{26}$ Body mass index was calculated as weight $(\mathrm{kg}) / \mathrm{height}(\mathrm{m})^{2}$. Adherence was assessed annually via a structured interview as well as weighing remaining pills. ${ }^{27}$ Depressive symptoms were measured using the Burnam screening algorithm, which consists of six items from the 20-item Center for Epidemiologic Studies Depression Scale and two items from the National Institute of Mental Health's Diagnostic Interview Schedule. ${ }^{28}$ The Burnam screen, initially developed for the National Study of Medical Care Outcomes, uses a logistic regression algorithm and provides a composite score between 0 and 1 . Using a cutpoint of 0.06 , this screening instrument has shown excellent sensitivity for detecting depressive disorder in the past month in primary care and mental health settings. ${ }^{28}$

\section{Data Analysis}

Proportional hazards regression was used to assess the differences in the distribution of time until the first occurrence of any impairment (i.e. either mild cognitive impairment or probable dementia), with covariate adjustment for treatment assignment in the other WHI trials. Censoring was defined by the last cognitive assessment. Differences in cognitive function test scores over time were assessed using general linear models ${ }^{29}$ with adjustment for baseline 3MSE, other WHI trial assignments, and other covariates as described below. To facilitate comparisons of associations among different cognitive domains, scores on tests were standardized by dividing the difference of individual scores from the cohort mean by the standard deviation.

\section{RESULTS}

A total of 4143 WHIMS participants were randomly assigned with equal probability to either combined calcium and vitamin D or placebo (Figure 1). These postmenopausal women had a mean age at screening of 71 years and an age range of 65 to 80 years (Table 1). There were no significant differences in age, ethnicity, education, medical co-morbidity, body mass index, alcohol consumption, physical activity or depression scores between treatment and placebo groups at baseline. There were no significant differences in assignments to other WHI trials or enrollment in WHISCA between placebo and treatment groups.

More women in the active treatment group were current or former smokers compared to the placebo group. A higher proportion of the placebo group reported past supplemental calcium and vitamin D use, but there were no significant differences in baseline dietary or total (dietary plus supplemental) calcium or vitamin D intake between groups. Baseline 25(OH)D 
levels did not differ between treatment and placebo groups in the subsample of women in whom these levels were obtained (mean level of $20.0 \mathrm{ng} / \mathrm{mL}( \pm 8.8)$ in treatment group, $\mathrm{N}=2034$ vs. $19.2 \mathrm{ng} / \mathrm{mL}( \pm 8.4)$ in placebo group, $\mathrm{N}=2109$; $\mathrm{p}=0.36$; see Table 1$)$. Women in the placebo group were slightly more adherent to calcium and vitamin D study medication than women in the treatment group $(75.5 \%$ were $\$ 80 \%$ adherent versus $72.3 \%$, respectively; $\mathrm{p}=0.02$ ). In contrast, there was no difference in mean adherence rates throughout follow-up between groups for hormone therapy medications.

As shown in Table 2, the incidence of cognitive impairment (probable dementia or MCI) did not differ significantly between placebo and treatment groups, with $4.8 \%$ of calcium plus vitamin D subjects (62.2 per 10,000 person years) and 5.1\% of placebo subjects (65.9 per 10,000 person years) developing cognitive impairment during a mean follow-up time of 7.8 years (hazard ratio $=0.94,95 \% \mathrm{CI}: 0.72-1.24, \mathrm{p}=0.68$ ). As seen in Figure 2, incidence of cognitive impairment was similar between treatment groups throughout follow-up. The incidence of dementia or MCI also did not differ significantly between groups after censoring follow-up time for women when adherence was $<80 \%$ (see Table 2). During follow-up, there were 39 cases of incident dementia among vitamin D and calcium subjects compared to 37 cases among placebo subjects (hazard ratio=1.11, 95\% CI: $0.71-1.74$, $\mathrm{p}=0.64$ ). Likewise, there were 98 cases of incident MCI among vitamin D subjects compared to 108 cases among placebo subjects (hazard ratio $=0.95,95 \% \mathrm{CI}: 0.72-1.25$, $\mathrm{p}=0.72$ ). During the course of follow-up, 16 women in the treatment group and 16 women in the control group were diagnosed with MCI at one visit and dementia at a separate visit.

The mean unadjusted 3MSE scores did not differ between placebo and treatment groups at baseline or in each of the 8 years following enrollment (Table 3). Mean scores on the 3MSE ranged from 95.4 to 96.7 , indicating well-preserved global cognitive function overall. After adjustment for several levels of covariates, ranging from a model including only baseline $3 \mathrm{MSE}$, smoking and year to a model including all of the baseline covariates in Table 1, there were no significant differences in 3MSE scores between treatment groups (results not shown).

Domain-specific measures of cognitive function were obtained in the 1,420 women who were enrolled in both the CaD trial and WHISCA and are shown in Table 4. These participants were seen for an average of $4.9(\mathrm{Ca} / \mathrm{Vit} \mathrm{D})$ and 5.0 (Placebo) annual visits through 2007. There were no significant differences in any domain-specific cognitive scores between treatment groups either in unadjusted analyses (not shown) or after adjustment for age, smoking, baseline 3MSE and treatment assignment in the hormone or diet modification trials.

\section{DISCUSSION}

In this large, randomized, double-blinded placebo-controlled trial, there was no effect of randomization to combined calcium and vitamin D versus placebo on incident cognitive impairment in elderly women. Women randomized to the combination of calcium and vitamin D or to placebo developed dementia or MCI at statistically similar rates over the long follow-up time of 7.8 years. Randomization also did not affect cognitive function on a global test or on seven domain-specific tests of cognitive function.

We are aware of no previous randomized controlled trials of calcium and vitamin D that examined cognitive outcomes. A recent interventional trial of vitamin D without calcium randomized 32 subjects with mild-to-moderate Alzheimer's disease to 6000 IU of vitamin $\mathrm{D}_{2}$ or placebo for 8 weeks (in addition to baseline $1000 \mathrm{IU}$ of $\mathrm{D}_{2}$ taken by all subjects for 8 week run-in and 8 week trial), followed immediately by randomization to intranasal insulin 
$60 \mathrm{IU}$ four times daily or placebo for 2 days. ${ }^{4}$ This study found no association between highdose vitamin $\mathrm{D}$ and changes in cognition or functioning.

Other studies of calcium and vitamin D and cognition have been observational, and the interpretation of these results has therefore been limited by the multiple potential confounders that can accompany these studies. Studies of vitamin D have generally demonstrated an association between low levels of 25(OH)D and increased odds of cognitive impairment in older adults, ${ }^{30-35}$ although some have found no evidence of an association. ${ }^{36-37}$ One population-based study of 5596 community-dwelling elderly women found that those with inadequate dietary vitamin D intake (defined as $<35 \mathrm{mcg} /$ week (approximately $200 \mathrm{IU} /$ day)) had lower mean cognitive scores and a greater likelihood of cognitive impairment than their counterparts with adequate vitamin D intake. ${ }^{38}$ Another study of 6257 community-dwelling elderly women found women with lower baseline $25(\mathrm{OH}) \mathrm{D}$ levels had higher risk of global cognitive decline but not decline of executive function. ${ }^{35}$ In a recent longitudinal study, 40 high-functioning older women were divided into two groups based on serum 25-OH-D levels (above or below $10 \mathrm{ng} / \mathrm{ml}$ ) at baseline..$^{39} 6$ women went on to develop non-Alzheimer's dementia over 7 years, and 4 women developed Alzheimer's. More non-Alzheimer's dementias were observed in women with vitamin D deficiency, while there was no between-group differences in the onset of AD. ${ }^{39}$

Regarding calcium, a prospective study of two independent population-based cohorts with 3994 subjects and 560 subjects, respectively, found an association between high serum calcium and poorer baseline global cognitive function as well as a greater rate of cognitive decline in people over the age of 75 , but not in younger subjects. ${ }^{10} \mathrm{~A}$ second study demonstrated that slightly elevated serum ionized calcium $(>1.29 \mathrm{mmol} / \mathrm{L})$ was associated with cognitive decline over ten years of follow-up in subjects with baseline ages of 75-85 years. ${ }^{9}$ A third study of women aged 75 years and older found no evidence of association between serum calcium levels and cognitive impairment. ${ }^{30}$ One way that calcium might increase the risk of cognitive impairment is via increasing risk of vascular cerebrovascular events. In a meta-analysis of 11 randomized controlled trials involving 12,000 subjects, calcium supplements were associated with a $30 \%$ increased risk of myocardial infarction, and with smaller nonsignificant increases in risk of stroke and mortality. ${ }^{40}$

There are several potential limitations to our study. Relatively few women converted to dementia in this study, so while there was sufficient power to detect a one-third lower risk for cognitive impairment in this study, power was limited for detecting smaller effects. The low rate of conversion to dementia is likely related to the relatively young age and the relatively high levels of baseline and maintained cognitive function of this population.

Women in this study were randomized to both vitamin $\mathrm{D}$ and calcium or to placebo, so it is difficult to determine what, if any, effect supplementation with vitamin $\mathrm{D}$ alone may have on cognition. In addition, the dose of vitamin D supplement of 400 IU per day was relatively low and may have not been enough to have an effect on cognitive function. Women in this study were generally not calcium or vitamin D deficient at the beginning of the intervention, so it is not possible to generalize the results of this study to women with calcium or vitamin D deficiency. Serum 25(OH)D levels were not measured later in the study, so we do not know how much the study supplementation affected serum levels.

Participants were able to take their own calcium and vitamin D supplements, which may have blunted any differences between the treatment groups. However, baseline dietary and total (dietary plus supplemental) calcium or vitamin D intake did not significantly differ between groups. In addition, a previously published comparison of 2-year post-enrollment serum 25(OH)D levels from 227 women randomized to calcium plus vitamin D versus 221 
women taking placebo in the WHI CaD trial found that the mean 25(OH)D level was $28 \%$ higher among women on active drug compared to the mean level of women assigned to placebo ${ }^{11}$, suggesting that the supplement dose and adherence rate was sufficient to cause a difference in serum vitamin D levels between groups. Non-adherence may have biased our results toward the null, but repeating our analyses after excluding non-adherent participants did not change our findings.

Women in the calcium and vitamin D treatment group were more likely to have been current or former smokers, and smoking has been associated with lower serum $25(\mathrm{OH}) \mathrm{D}$ and $1,25(\mathrm{OH}) 2 \mathrm{D}$ levels. ${ }^{41}$ However, there was less than a $5 \%$ difference in smoking rates between groups ( $51.8 \%$ of women in the calcium and vitamin $\mathrm{D}$ group smoked compared to $56.3 \%$ in the placebo group), and even after adjusting for smoking in the models, there was no difference in outcomes between placebo and treatment groups.

Participants were exclusively women, which may limit the generalizability of these findings to men, and were primarily non-Hispanic Caucasian, which may limit the generalizability to other ethnic groups.

In summary, there was no association between treatment assignment and incident dementia or MCI or cognitive function in this large prospective randomized trial of calcium and vitamin D in elderly women. Further investigations are needed to investigate the association between calcium and vitamin $\mathrm{D}$ and cognition in men and in other age and ethnic groups, and to examine the effects of calcium and vitamin D separately, especially higher doses of vitamin D without calcium supplementation among persons with low serum levels of vitamin D.

\section{Acknowledgments}

The authors thank the WHI investigators and staff for their dedication, and the study participants for making the program possible. A listing of WHI investigators can be found at: http://www.whiscience.org/publications/ WHI_investigators_shortlist.pdf.

Funding/Support: The WHI program is funded by the National Heart, Lung, and Blood Institute, National Institutes of Health, U.S. Department of Health and Human Services through contracts N01WH22110, 24152, 32100-2, 32105-6, 32108-9, 32111-13, 32115, 32118-32119, 32122, 42107-26, 42129-32, and 44221.

Dr. Rossom's work on this manuscript was supported in part by VHA through Clinical and Health Services Research and Development Grants 04SCRCOE-001 and HFP-98-001.

Dr. JoAnn Manson and colleagues at Brigham and Women's Hospital, Harvard Medical School, are recipients of funding from the National Institutes of Health to conduct the VITamin D and OmegA-3 TriaL (VITAL), a largescale randomized trial of vitamin D and omega-3s in the prevention of cancer and cardiovascular disease.

Sponsor's Role: The sponsors did not play any role in the design or conduct of the study; collection, management, analysis, or interpretation of the data; or preparation, review, or approval of the manuscript.

\section{References}

1. Grant WB. Does vitamin D reduce the risk of dementia? J Alzheimers Dis. 2009; 17:151-159. [PubMed: 19494440]

2. Moore ME, Piazza A, McCartney Y, et al. Evidence that vitamin D3 reverses age-related inflammatory changes in the rat hippocampus. Biochem Soc Trans. 2005; 33:573-577. [PubMed: 16042547]

3. Obradovic D, Gronemeyer H, Lutz B, et al. Cross-talk of vitamin D and glucocorticoids in hippocampal cells. J Neurochem. 2006; 96:500-509. [PubMed: 16336217] 
4. Stein MS, Scherer SC, Ladd KS, et al. A randomized controlled trial of high-dose vitamin D2 followed by intranasal insulin in Alzheimer's disease. J Alzheimers Dis. 2011; 26:477-484. [PubMed: 21694461]

5. Annweiler C, Allali G, Allain P, et al. Vitamin D and cognitive performance in adults: A systematic review. Eur J Neurol. 2009; 16:1083-1089. [PubMed: 19659751]

6. Fedrizzi L, Carafoli E. Ca2+ dysfunction in neurodegenerative disorders: Alzheimer's disease. Biofactors. 2011; 37:189-196. [PubMed: 21698698]

7. Bojarski L, Herms J, Kuznicki J. Calcium dysregulation in Alzheimer's disease. Neurochem Int. 2008; 52:621-633. [PubMed: 18035450]

8. Green KN, LaFerla FM. Linking calcium to Abeta and Alzheimer's disease. Neuron. 2008; 59:190194. [PubMed: 18667147]

9. Tilvis RS, Kahonen-Vare MH, Jolkkonen J, et al. Predictors of cognitive decline and mortality of aged people over a 10-year period. J Gerontol A Biol Sci Med Sci. 2004; 59:268-274. [PubMed: 15031312]

10. Schram MT, Trompet S, Kamper AM, et al. Serum calcium and cognitive function in old age. J Am Geriatr Soc. 2007; 55:1786-1792. [PubMed: 17979900]

11. Jackson RD, LaCroix AZ, Gass M, et al. Calcium plus vitamin D supplementation and the risk of fractures. N Engl J Med. 2006; 354:669-683. [PubMed: 16481635]

12. Wactawski-Wende J, Kotchen JM, Anderson GL, et al. Calcium plus vitamin D supplementation and the risk of colorectal cancer. N Engl J Med. 2006; 354:684-696. [PubMed: 16481636]

13. Shumaker SA, Legault C, Rapp SR, et al. Estrogen plus progestin and the incidence of dementia and mild cognitive impairment in postmenopausal women: The Women's Health Initiative Memory Study: A randomized controlled trial. JAMA. 2003; 289:2651-2662. [PubMed: 12771112]

14. Shumaker SA, Reboussin BA, Espeland MA, et al. The Women's Health Initiative Memory Study (WHIMS): A trial of the effect of estrogen therapy in preventing and slowing the progression of dementia. Control Clin Trials. 1998; 19:604-621. [PubMed: 9875839]

15. Stefanick ML, Cochrane BB, Hsia J, et al. The Women's Health Initiative postmenopausal hormone trials: Overview and baseline characteristics of participants. Ann Epidemiol. 2003; 13(9 Suppl):S78-86. [PubMed: 14575940]

16. Ritenbaugh C, Patterson RE, Chlebowski RT, et al. The women's health initiative dietary modification trial: Overview and baseline characteristics of participants. Ann Epidemiol. 2003; 13(9, Supplement 1):S87-S97. [PubMed: 14575941]

17. Shumaker SA, Legault C, Kuller L, et al. Conjugated equine estrogens and incidence of probable dementia and mild cognitive impairment in postmenopausal women: Women's Health Initiative Memory Study. JAMA. 2004; 291:2947-2958. [PubMed: 15213206]

18. Espeland MA, Rapp SR, Shumaker SA, et al. Conjugated equine estrogens and global cognitive function in postmenopausal women: Women's Health Initiative Memory Study. JAMA. 2004; 291:2959-2968. [PubMed: 15213207]

19. Resnick SM, Coker LH, Maki PM, et al. The Women's Health Initiative Study of Cognitive Aging (WHISCA): A randomized clinical trial of the effects of hormone therapy on age-associated cognitive decline. Clin Trials. 2004; 1:440-450. [PubMed: 16279282]

20. Wactawski-Wende J, Kotchen JM, Anderson GL, et al. Calcium plus vitamin D supplementation and the risk of colorectal cancer. N Engl J Med. 2006; 354:684-696. [PubMed: 16481636]

21. American Psychiatric A. Diagnostic and Statistical Manual of Mental Disorders. Vol. 4. Washington, D.C: American Psychiatric Association; 1994.

22. Morris JC, Heyman A, Mohs RC, et al. The Consortium to Establish a Registry for Alzheimer's Disease (CERAD). Part I. Clinical and neuropsychological assessment of Alzheimer's disease. Neurology. 1989; 39:1159-1165. [PubMed: 2771064]

23. Petersen RC, Doody R, Kurz A, et al. Current concepts in mild cognitive impairment. Arch Neurol. 2001; 58:1985-1992. [PubMed: 11735772]

24. Shumaker SA, Legault C, Kuller L, et al. Conjugated Equine Estrogens and Incidence of Probable Dementia and Mild Cognitive Impairment in Postmenopausal Women. JAMA 2004. 2004; 291:2947-2958. 
25. Teng EL, Chui HC. The Modified Mini-Mental State (3MS) examination. J Clin Psychiatry. 1987; 48:314-318. [PubMed: 3611032]

26. Wilcock GK, Lilienfeld S, Gaens E. Efficacy and safety of galantamine in patients with mild to moderate Alzheimer's disease: Multicentre randomised controlled trial. Galantamine International-1 Study Group. BMJ. 2000; 321:1445-1449. [PubMed: 11110737]

27. Brunner R, Dunbar-Jacob J, Leboff MS, et al. Predictors of adherence in the Women's Health Initiative Calcium and Vitamin D Trial. Behav Med. 2009; 34:145-155. [PubMed: 19064373]

28. Burnam MA, Wells KB, Leake B, et al. Development of a brief screening instrument for detecting depressive disorders. Med Care. 1988; 26:775-789. [PubMed: 3398606]

29. Littell, RC.; Milliken, GA.; Stroup, WW., et al. SAS System for Mixed Models. Cary, NH: SAS Institute Ince; 1996.

30. Annweiler C, Schott AM, Allali G, et al. Association of vitamin D deficiency with cognitive impairment in older women: Cross-sectional study. Neurology. 2010; 74:27-32. [PubMed: 19794127]

31. Llewellyn DJ, Langa KM, Lang IA. Serum 25-hydroxyvitamin D concentration and cognitive impairment. J Geriatr Psychiatry Neurol. 2009; 22:188-195. [PubMed: 19073839]

32. Buell JS, Scott TM, Dawson-Hughes B, et al. Vitamin D is associated with cognitive function in elders receiving home health services. J Gerontol A Biol Sci Med Sci. 2009; 64:888-895. [PubMed: 19377013]

33. Lee DM, Tajar A, Ulubaev A, et al. Association between 25-hydroxyvitamin D levels and cognitive performance in middle-aged and older European men. J Neurol Neurosurg Psychiatry. 2009; 80:722-729. [PubMed: 19460797]

34. Dickens AP, Lang IA, Langa KM, et al. Vitamin D, cognitive dysfunction and dementia in older adults. CNS Drugs. 2011; 25:629-639. [PubMed: 21790207]

35. Slinin Y, Paudel M, Taylor BC, et al. Association between serum $25(\mathrm{OH})$ vitamin D and the risk of cognitive decline in older women. J Gerontol A Biol Sci Med Sci. 2012 Mar 27. [Epub ahead of print].

36. Slinin Y, Paudel ML, Taylor BC, et al. 25-Hydroxyvitamin D levels and cognitive performance and decline in elderly men. Neurology. 2010; 74:33-41. [PubMed: 19940271]

37. Jorde R, Waterloo K, Saleh F, et al. Neuropsychological function in relation to serum parathyroid hormone and serum 25-hydroxyvitamin D levels. The Tromso study. J Neurol. 2006; 253:464470. [PubMed: 16283099]

38. Annweiler C, Schott AM, Rolland Y, et al. Dietary intake of vitamin D and cognition in older women: A large population-based study. Neurology. 2010; 75:1810-1816. [PubMed: 21079183]

39. Annweiler C, Rolland Y, Schott AM, et al. Serum vitamin D deficiency as a predictor of incident non-Alzheimer dementias: A 7-year longitudinal study. Dement Geriatr Cogn Disord. 2011; 32:273-278. [PubMed: 22261995]

40. Bolland MJ, Avenell A, Baron JA, et al. Effect of calcium supplements on risk of myocardial infarction and cardiovascular events: Meta-analysis. BMJ. 2010; 341:c3691. [PubMed: 20671013]

41. Brot C, Jorgensen NR, Sorensen $\mathrm{OH}$. The influence of smoking on vitamin D status and calcium metabolism. Eur J Clin Nutr. 1999; 53:920-926. [PubMed: 10602348] 


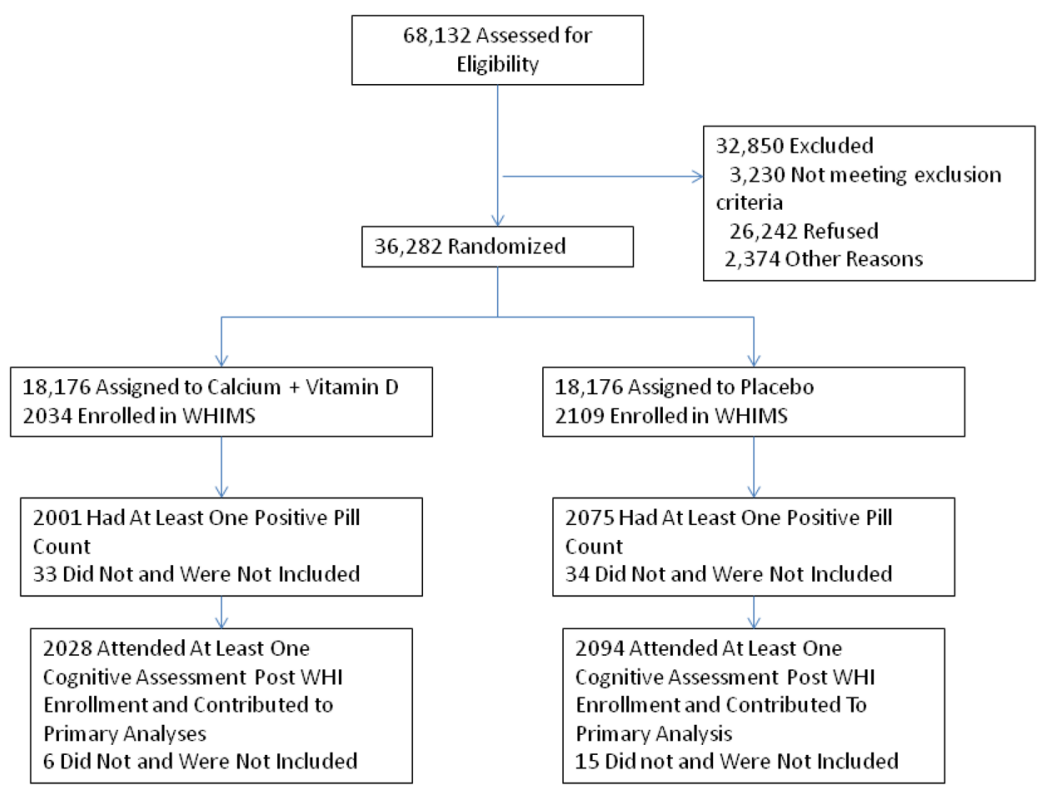

Figure 1.

Study Flow Diagram. 


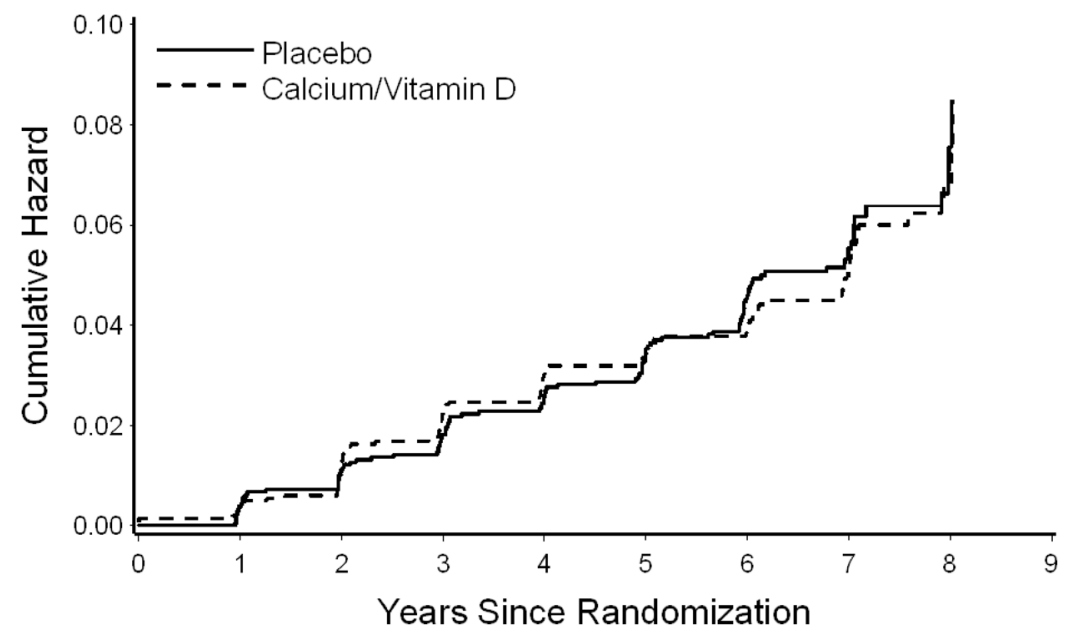

Figure 2. Incidence of Mild Cognitive Impairment or Probable Dementia: All Women by Treatment Assignment (Placebo versus Calcium/Vitamin D)* $* \mathrm{p}=0.68$ 
Table 1

Baseline Characteristics of Participants Grouped by Treatment Assignment.

\begin{tabular}{|c|c|c|c|}
\hline Characteristics & Calcium/Vitamin D N=2034 & Placebo $N=2109$ & P-value \\
\hline \multicolumn{4}{|l|}{ Age at screening, years } \\
\hline Mean age & 70.7 & 70.9 & 0.22 \\
\hline $65-69$ & $1003(49.4 \%)$ & $984(46.7 \%)$ & \\
\hline $70-74$ & $692(34.1 \%)$ & $774(36.7 \%)$ & 0.16 \\
\hline $75-80$ & $337(16.6 \%)$ & $351(16.6 \%)$ & \\
\hline \multicolumn{4}{|l|}{ Race/ethnicity } \\
\hline American Indian/Alaskan & $9(0.4 \%)$ & $7(0.3 \%)$ & \\
\hline \multicolumn{4}{|l|}{ Native } \\
\hline Asian/Pacific Islander & $37(1.8 \%)$ & $38(1.8 \%)$ & \\
\hline Black/African-American & $128(6.3 \%)$ & $121(5.7 \%)$ & \\
\hline Hispanic/Latino & $41(2.0 \%)$ & $59(2.8 \%)$ & 0.61 \\
\hline White, non-Hispanic & $1792(88.2 \%)$ & $1859(88.2 \%)$ & \\
\hline Other & $26(1.3 \%)$ & $25(1.2 \%)$ & \\
\hline \multicolumn{4}{|l|}{ Education } \\
\hline Not high school grad & $141(7.0 \%)$ & $146(6.9 \%)$ & \\
\hline High school grad & $444(21.9 \%)$ & $469(22.2 \%)$ & 0.98 \\
\hline Post-HS & $812(40.0 \%)$ & $847(40.2 \%)$ & \\
\hline College graduate & $633(31.2 \%)$ & $647(30.7 \%)$ & \\
\hline \multicolumn{4}{|l|}{ Medical co-morbidity } \\
\hline History of myocardial infarction & $63(3.1 \%)$ & $68(3.2 \%)$ & 0.82 \\
\hline History of stroke or TIA & $75(3.7 \%)$ & $69(3.3 \%)$ & 0.47 \\
\hline Diabetes & $148(7.3 \%)$ & $167(7.9 \%)$ & 0.43 \\
\hline Hypertension & $986(48.5 \%)$ & $1029(48.8 \%)$ & 0.84 \\
\hline \multicolumn{4}{|l|}{ Body mass index, $\mathrm{kg} / \mathrm{m}^{2}$} \\
\hline$<25.0$ & $565(28.0 \%)$ & $599(28.5 \%)$ & \\
\hline $25.0-29.9$ & $729(36.1 \%)$ & $741(35.3 \%)$ & 0.84 \\
\hline$\geq 30.0$ & $724(35.9 \%)$ & $761(36.2 \%)$ & \\
\hline \multicolumn{4}{|l|}{ Cigarette smoking } \\
\hline Never & $1039(51.8 \%)$ & $1174(56.3 \%)$ & \\
\hline Former & $809(40.4 \%)$ & $784(37.6 \%)$ & 0.007 \\
\hline Current & $156(7.8 \%)$ & $127(6.1 \%)$ & \\
\hline \multicolumn{4}{|l|}{ Alcohol use, drinks/day } \\
\hline None & $946(46.6 \%)$ & $964(45.8)$ & \\
\hline$\underline{2}$ & $990(48.8 \%)$ & $1061(50.4)$ & 0.36 \\
\hline$>2$ & $92(4.5 \%)$ & $80(3.8)$ & \\
\hline
\end{tabular}




\begin{tabular}{|c|c|c|c|}
\hline Characteristics & Calcium/Vitamin D N=2034 & Placebo $N=2109$ & P-value \\
\hline \multicolumn{4}{|c|}{ Burnam score for depressive symptoms } \\
\hline$\unlhd) .06$ & $1880(92.4 \%)$ & $1953(92.6 \%)$ & 0.83 \\
\hline$>0.06$ & $154(7.6 \%)$ & $156(7.4 \%)$ & \\
\hline \multicolumn{4}{|c|}{ Recreational physical activity, METS/week } \\
\hline $0-3.5$ & $657(32.4 \%)$ & $674(32.0 \%)$ & \\
\hline $3.6-12.5$ & $673(33.2 \%)$ & $729(34.6 \%)$ & 0.59 \\
\hline$\geq 12.5$ & $699(34.4 \%)$ & $702(33.4 \%)$ & \\
\hline \multicolumn{4}{|l|}{ Supplement use } \\
\hline Calcium & $1061(52.2 \%)$ & $1196(56.7 \%)$ & 0.003 \\
\hline Vitamin D & $929(45.7 \%)$ & $1047(49.6 \%)$ & 0.01 \\
\hline \multicolumn{4}{|c|}{ Dietary calcium intake, mg/day* } \\
\hline$<600$ & $686(38.7 \%)$ & $702(38.0 \%)$ & \\
\hline $600-799$ & $359(20.2 \%)$ & $351(19.0 \%)$ & 0.61 \\
\hline $800-1199$ & $447(25.2 \%)$ & $480(26.0 \%)$ & \\
\hline$\geq 1200$ & $281(15.8 \%)$ & $315(17.0 \%)$ & \\
\hline \multicolumn{4}{|c|}{ Total calcium intake (supplements + diet), mg/day } \\
\hline$<600$ & $423(23.9 \%)$ & $435(23.5 \%)$ & \\
\hline $600-799$ & $259(14.6 \%)$ & $241(13.0 \%)$ & 0.26 \\
\hline $800-1199$ & $463(26.1 \%)$ & $464(25.1 \%)$ & \\
\hline$\geq 1200$ & $628(35.4 \%)$ & $708(38.3 \%)$ & \\
\hline \multicolumn{4}{|c|}{ Dietary vitamin D intake, IU/day } \\
\hline$<200$ & $380(21.4 \%)$ & $355(19.2 \%)$ & \\
\hline 200-399 & $594(33.5 \%)$ & $646(35.0 \%)$ & 0.08 \\
\hline $400-599$ & $444(25.0 \%)$ & $430(23.3 \%)$ & \\
\hline 2600 & $355(20.0 \%)$ & $417(22.6 \%)$ & \\
\hline \multicolumn{4}{|c|}{ Total vitamin D intake (supplements + diet), IU/day } \\
\hline$<200$ & $256(14.4 \%)$ & $227(12.3 \%)$ & \\
\hline 200-399 & $213(12.0 \%)$ & $197(10.7 \%)$ & 0.09 \\
\hline $400-599$ & $331(18.7 \%)$ & $348(18.8 \%)$ & \\
\hline$\checkmark 600$ & $973(54.9 \%)$ & $1076(58.2 \%)$ & \\
\hline \multicolumn{4}{|c|}{ Dietary modification trial assignment } \\
\hline Control & $306(15.0 \%)$ & $301(14.3 \%)$ & \\
\hline Intervention & $178(8.8 \%)$ & $228(10.8 \%)$ & 0.08 \\
\hline Not in trial & $1550(76.2 \%)$ & $1580(74.9 \%)$ & \\
\hline \multicolumn{4}{|l|}{ HT treatment assignment } \\
\hline CEE-alone placebo & $385(18.9 \%)$ & $415(19.7 \%)$ & \\
\hline CEE-alone & $396(19.5 \%)$ & $367(17.4 \%)$ & 0.06 \\
\hline CEE+MPA placebo & $587(28.9 \%)$ & $677(32.1 \%)$ & \\
\hline
\end{tabular}




\begin{tabular}{|c|c|c|c|}
\hline Characteristics & Calcium/Vitamin D N=2034 & Placebo $N=2109$ & P-value \\
\hline CEE+MPA & $666(32.7 \%)$ & $650(30.8 \%)$ & \\
\hline \multicolumn{4}{|c|}{ Adherence to HT study drug $b$} \\
\hline$<80 \%$ adherent & $500(24.6 \%)$ & $507(24.1 \%)$ & 0.67 \\
\hline$\searrow 80 \%$ adherent & $1529(75.4 \%)$ & $1599(75.9 \%)$ & \\
\hline \multicolumn{4}{|c|}{ Adherence to CaD study drug $b$} \\
\hline$<80 \%$ adherent & $555(27.7 \%)$ & $509(24.5 \%)$ & 0.02 \\
\hline$₫ 80 \%$ adherent & $1446(72.3 \%)$ & $1566(75.5 \%)$ & \\
\hline \multicolumn{4}{|l|}{ WHISCA enrollment } \\
\hline No & $1344(66.1 \%)$ & $1379(65.4 \%)$ & 0.64 \\
\hline Yes & $690(33.9 \%)$ & $730(34.6 \%)$ & \\
\hline \multicolumn{4}{|c|}{ Baseline 25(OH)D level, ng/mL } \\
\hline Sample size & $\mathrm{N}=150$ & $\mathrm{~N}=143$ & \\
\hline Mean level $( \pm$ SD) & $20.0( \pm 8.8)$ & $19.2( \pm 8.4)$ & $0.36^{c}$ \\
\hline Tertile $1(<15.0)$ & $49(32.7 \%)$ & $49(34.3 \%)$ & \\
\hline Tertile $2(15.0-22.5)$ & $52(34.7 \%)$ & $46(32.2 \%)$ & 0.90 \\
\hline Tertile 3 (>22.5) & $49(32.7 \%)$ & $48(33.6 \%)$ & \\
\hline
\end{tabular}

${ }^{a}$ Data are expressed as number (percentage) unless otherwise specified. Numbers across categories may not sum to the given totals because of missing data.

${ }^{b}$ Mean adherence throughout follow-up.

$c_{\text {Based on log-transformed levels. }}$ 
Table 2

Incidence of Probable Dementia or Mild Cognitive Impairment (MCI) by Treatment Assignment

\begin{tabular}{|c|c|c|c|}
\hline & Calcium/Vitamin D N=2034 & Placebo $N=2109$ & HR $(95 \%$ CI $)$ \\
\hline \multicolumn{4}{|l|}{ Intent-to Treat Analysis } \\
\hline Probable Dementia or Mild Cognitive Impairment, No. (\%) & $98(4.8 \%)$ & $108(5.1 \%)$ & \multirow{3}{*}{$\begin{array}{c}0.94(0.72,1.24) \\
P=0.68\end{array}$} \\
\hline Follow-up, mean, years $( \pm \mathrm{SD})$ & $7.7( \pm 2.5)$ & $7.8( \pm 2.5)$ & \\
\hline Rate per 10,000 person-years & 62.2 & 65.9 & \\
\hline \multicolumn{4}{|c|}{ Analysis Excluding Follow-Up Time for Non-Adherent Participants ${ }^{a}$} \\
\hline Probable Dementia or Mild Cognitive Impairment, No. (\%) & $62(3.1 \%)$ & $62(2.9 \%)$ & \multirow{3}{*}{$\begin{array}{c}1.05(0.74,1.49) \\
\mathrm{P}=0.79\end{array}$} \\
\hline Follow-up, mean, years $( \pm \mathrm{SD})$ & $6.4( \pm 1.7)$ & $6.44( \pm 1.6)$ & \\
\hline Rate per 10,000 person-years & 48.0 & 45.7 & \\
\hline
\end{tabular}

${ }^{a}$ Participants excluded 6 months after nonadherence detected 


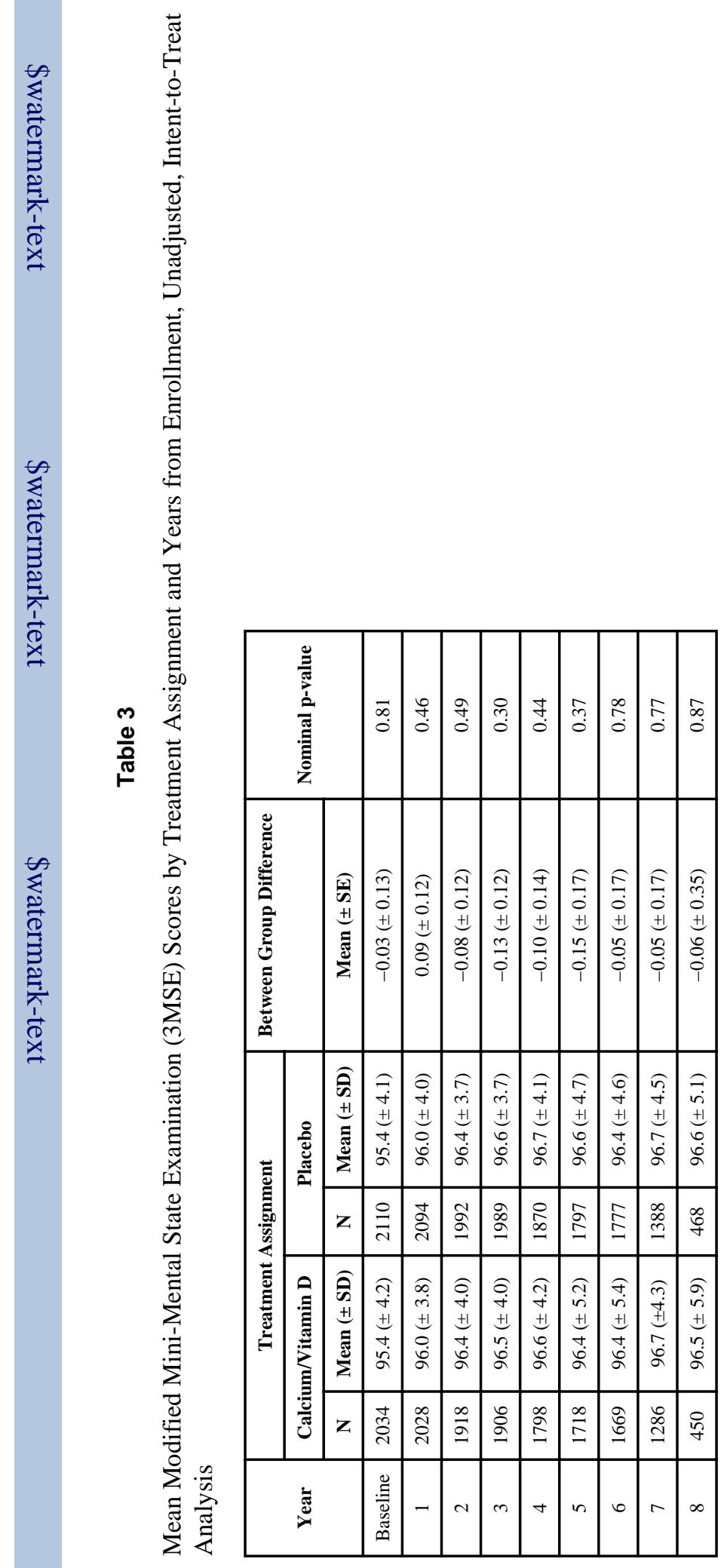

J Am Geriatr Soc. Author manuscript; available in PMC 2013 December 01. 


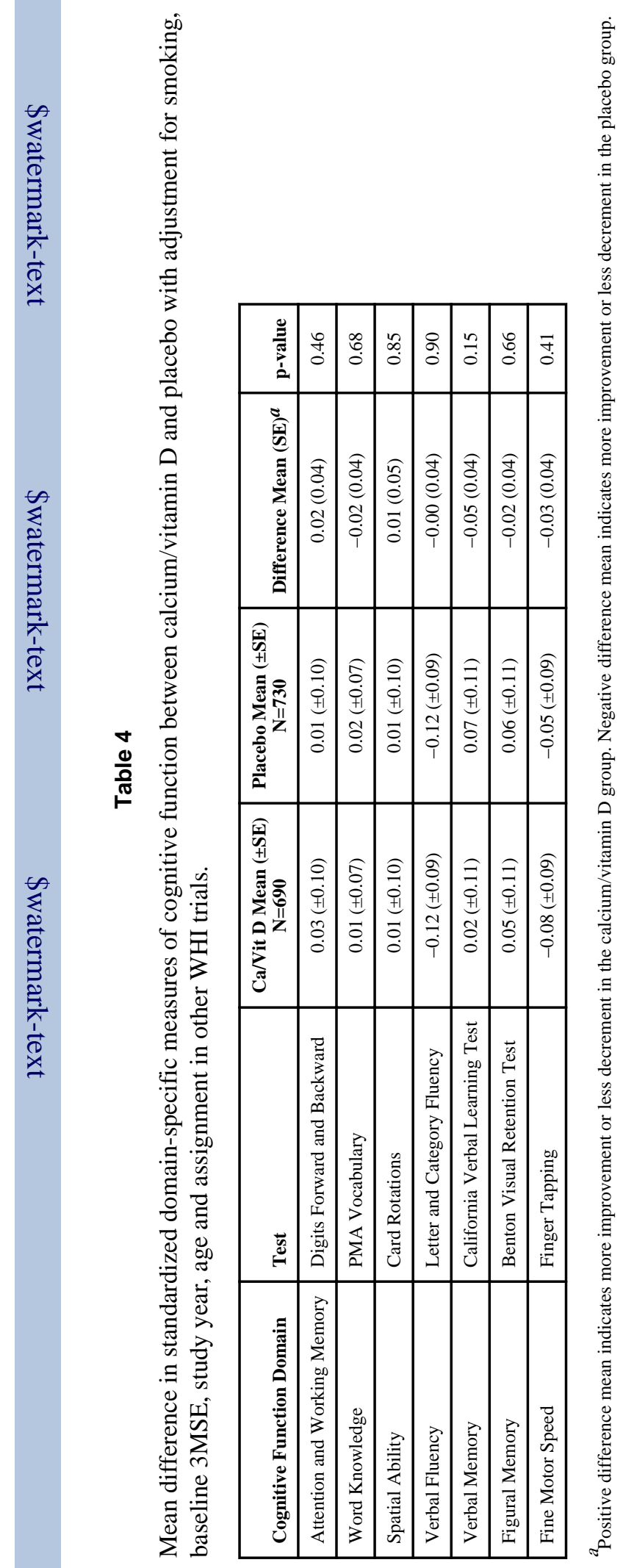

